\title{
NONLINEAR DYNAMICS OF IMMUNOGENIC TUMORS: PARAMETER ESTIMATION AND GLOBAL BIFURCATION ANALYSIS
}

\author{
- Vladimir A. Kuznetsov and Iliya A. Makalkin \\ Laboratory of Mathematical Immunobiophysics, \\ Institute of Chemical Physics, \\ Russian Academy of Sciences, \\ Kosygin str. 4, bldg. 8, \\ Moscow GSP-1, 117977 Russia \\ (E.mail: chembio@glas.apc.org) \\ - Mark A. Taylor and alan S. Perelson \\ Theoretical Division, \\ Los Alamos National Laboratory, \\ Los Alamos, NM 87545, U.S.A. \\ (E.mail: asp@receptor.lanl.gov)
}

\begin{abstract}
We present a mathematical model of the cytotoxic $\mathrm{T}$ lymphocyte response to the growth of an immunogenic tumor. The model exhibits a number of phenomena that are seen in vivo, including immunostimulation of tumor growth, "sneaking through" of the tumor, and formation of a tumor "dormant state". The model is used to describe the kinetics of growth and regression of the B-lymphoma $\mathrm{BCL}_{1}$ in the spleen of mice. By comparing the model with experimental data, numerical estimates of parameters describing processes that cannot be measured in vivo are derived. Local and global bifurcations are calculated for realistic values of the parameters. For a large set of parameters we predict that the course of tumor growth and its clinical manifestation have a recurrent profile with a 3 - to 4 -month cycle, similar to patterns seen in certain leukemias.
\end{abstract}

1. Introduction. The immune response to a tumor is usually cell-mediated with cytotoxic $\mathrm{T}$ lymphocytes (CTL) and natural killer (NK) cells playing a dominant role. A number of mathematical models of the interactions between the immune system and a growing tumor have been developed (Thorn and Henney, 1976, 1977; DeLisi and Rescigno, 1977; Rescigno and DeLisi, 1977; Kuznetsov and Volkenshtein, 1978, 1979; Albert et al., 1980; Prigogine and Lefever, 1980; Look et al., 1981; Lefever and Erneaux, 1984; Grossman and Berke, 1980; De Boer and Hogeweg, 1985, 1986; Perelson and Macken, 1984; Hiernaux et al., 1986; Merrill and Sathananthan, 1986; Mohler and Lee, 1989; Dozmorov and Kuznetsov, 1988). The kinetics of cell mediated cytotoxicity in vitro have also been described by mathematical models (Thorn and Henney, 1976, 1977; Thoma et al., 1978; Merrill, 1982; Perelson and Bell, 1982; Perelson and Macken, 1984; Macken and Perelson, 1984; Callewaert et al., 1988; 
Kuznetsov, 1979, 1981, 1984; Lefever et al., 1992). With such models, numerical estimates of biologically significant parameters have been obtained, a number of phenomena interpreted, and predictions made.

The dynamics of the anti-tumor immune response in vivo are complicated and are not well understood. Spontaneously arising tumors are known to be of low immunogenicity and usually grow out of control in an organism. The escape from immune surveillance has been linked with a number of different mechanisms, including the selection of tumor clones resistant to cytolytic mechanisms, the loss or masking of tumor antigens, the loss of MHC class I molecules, and tumor induced disorders in immunoregulation (Brondz, 1987; Nelson and Nelson, 1987; Tanaka et al., 1988). Nevertheless, cancer cells are attacked and killed by cells of the immune system (Hellström and Hellström, 1969; Herberman, 1974; Greenberg, 1991), and thus immune surveillance of spontaneous tumors may be effective and important in keeping cancer incidence low.

The main attempts at present to develop schemes for immunotherapy or its combination with other therapy methods are directed at lowering tumor mass, heightening tumor immunogenicity, and removal of immunosuppression induced in an organism in the process of tumor growth. Nevertheless, the majority of such attempts are not effective. One of the main reasons for this lies in the fact that even after a so-called "successful" and "clinically" complete removal of a tumor, a small quantity of "residual" tumor cells stay in an organism, which can grow into secondary tumors or "dormant" metastases (Mathe and Rejzenstein, 1986; Wheelock and Robinson, 1983; Yefenof et al., 1993).

Tumor dormancy is an operational term used to describe a state in which potentially lethal tumor cells persist for a prolonged period of time with little or no increase in the tumor cell population (Wheelock et al., 1983; Stewart and Wheelock, 1992; Yefenof et al., 1993). It is frequently presumed that tumor cells do not grow at a rapid rate during dormancy, say due to the absence of a factor needed for progressive growth into a tumor, but an alternative possibility is that rapidly growing cells are killed at a rate equal to that at which they generated (Uhr et al., 1991). Dormant states emerge not only after a radical treatment of a tumor, but also at early stages of tumor progression. In fact, there is general agreement that in the human, neoplastic cells escape from a primary tumor very early in its development. The fate of these escaping neoplastic cells will determine whether the patient lives or dies of cancer (Uhr et al., 1991). The direct participation of CTL in the support of a tumor dormant state has been shown in some experimental models (Wheelock and Robinson, 1983; Weinhold et al., 1979a,b). Besides CTL, other types of immune system cells, such as macrophages and NK-cells, may participate in the maintenance of a tumor dormant state. Anti-idiotype antibodies may also play a role in inducing 
the tumor cells into a dormant state (Uhr et al., 1991). Although considerable research has been carried out, our understanding of the mechanisms of immune surveillance and of the tumor dormant state is still quite incomplete (Stewart and Wheelock, 1992; Chen et al., 1990; Liu et al., 1990).

Small dormant tumors, which after a long time begin uncontrolled growth, may escape from immune surveillance by the so-called "sneaking through mechanism" (Uyttenhove et al., 1983; Wheelock et al., 1981; Wheelock and Robinson, 1983). Sneaking through refers to a phenomenon in which animals, when challenged with a low dose of tumor cells fail to mount a successful antitumor immune response and progressive tumor growth results; challenge with medium doses of tumor cells leads to tumor rejection, and large doses break through the immune defenses and successfully generate tumors (Old et al., 1962; Deichman, 1979). This effect has been reproduced in different experimental models (Deichman, 1979; Gatenby et al., 1981). Experimentally, "sneaking through" can be most easily manifested after a preliminary immunization with a subimmunogenic dose of tumor antigens (Gatenby et al., 1981; Deichman et al., 1979). The time between initial immunization and injection of tumor cells is an important parameter (Alsabti, 1978; Deichman, 1979; Deichman et al., 1979). The mechanisms responsible for sneaking through are not known and the phenomenon has been the subject of a variety of mathematical models (DeLisi and Rescigno, 1977; Rescigno and DeLisi, 1977; Grossman and Berke, 1980; De Boer and Hogeweg, 1985, 1986).

Different host defense cells are able to suppress the growth of or destroy tumor cells. However, in a number of experimental and clinical cases it has been observed that stimulation of the immune system by immunotherapy results in the stimulation of tumor cell growth rather than suppression (Prehn, 1983; Colmeraver et al., 1980; Sampson et al., 1977). There are even cases of human or animal tumors being stimulated by the very lymphoid cells which may also cause their lysis. For example, immune CTL in small amounts have been shown to stimulate tumor cell population growth in vitro (Fidler, 1973; Jeejeebhoy, 1977), and in vivo after an injection of immune lymphocytes and tumor cells into lethally irradiated animals (Prehn, 1972, 1983; Umiel and Trainin, 1974). Accelerated growth of an allogeneic tumor in an animal first immunized against this tumor has been observed, immune $\mathrm{T}$ lymphocytes being responsible for this effect (Prehn, 1983). The mechanisms responsible for immunostimulation of tumor growth are not clear, but the very fact of their existence restrains the carrying out of immunotherapy.

There are different explanations for the termination of a tumor dormant state, for sneaking through of tumors, and for immunostimulation effects. Often these explanations are based on the ideas of immunoselection, antigenic modulation, production by tumor cells of different types of immune cell blocking factors, generation of immunosuppressor cells, changes in auto- 
regulatory networks in a tumor localization region, and other more complex ideas that are very difficult to prove or disprove experimentally. Here we propose that these phenomena may be the result of nonlinear dynamic interactions between the tumor and the immune system (also see Kuznetsov, 1984, 1987, 1988, 1991).

In this paper, we analyse a simple mathematical model of a cell mediated response to a growing tumor cell population. This model differs from most others in the literature in that it takes into account the infiltration of the tumor by effector cells as well as the possibility of effector cell inactivation. A variant of this model has been studied by Kuznetsov (1991). Here we focus on the qualitative behavior of the system using techniques from bifurcation theory. We apply the model to the analysis of the mechanisms of tumor dormancy and sneaking through. Interestingly, we find that a non-zero rate of effector cell inactivation is required to obtain sneaking through. We also find that sneaking through, tumor dormancy and the immunostimulation of tumor growth, effects which have been analysed separately, according to our model, may all be related.

2. Mathematical Model. It has been found in numerous studies both in vivo and in vitro that the growth of a tumor cell population is exponential for small quantities of tumor cells but growth is slowed at large population sizes. The inhibition of growth may be caused by the competition of cells for metabolites and/or growth factors, or by growth inhibiting factors produced by the tumor cells. In many cases of non-exponential tumor growth, the kinetics are well described by the logistic or Gompertz equation (Emanuel, 1981; Swan, 1977).

Consider a tumor whose cells are "immunogenic", and thus subject to immune attack by cytotoxic effector cells, e.g. CTL or NK cells. The interaction between effector cells (EC) and tumor cells (TC) in vitro can be described by the kinetic scheme:

$$
E+T \underset{k_{-1}}{\stackrel{k_{-1}}{\rightleftarrows}} C \underset{k_{3}}{\stackrel{k_{2}}{\rightleftarrows}} E+T^{*}+T
$$

where $E, T, C, E^{*}, T^{*}$ are the local concentrations of effector cells, tumor cells, effector cell-tumor cell conjugates, inactivated effector cells, and "lethally hit" TC cells, respectively. Lethally hit tumor cells are destined to perish. They also have been called cells "programmed to die". The inclusion of inactivated effector cells is an unusual feature of our model. NK cells, and to a lesser extent CTL, in culture seem to have a limited ability to repeatedly kill target cells (Abrahms and Brahmi, 1988; Callewaert et al., 1988; Kuznetsov et al., 1988). This might be due to exhaustion of molecules responsible for the cytotoxic effect or other regulatory effects, possibly due to the release of molecules from 
the tumor cell when the TC and EC are conjugated. The parameters $k_{1}, k_{-1}, k_{2}$ and $k_{3}$ are non-negative kinetic constants: $k_{1}$ and $k_{-1}$ describe the rates of binding of $\mathrm{EC}$ to TC and detachment of $\mathrm{EC}$ from TC without damaging cells; $k_{2}$ is the rate at which EC-TC interactions irreversibly program TC for lysis; and $k_{3}$ is the rate at which EC-TC interactions inactivate EC.

We propose the following system of differential equations as a model for the interaction between $\mathrm{EC}$ and a growing immunogenic tumor in vivo:

$$
\begin{aligned}
\frac{\mathrm{d} E}{\mathrm{~d} t} & =s+F(C, T)-d_{1} E-k_{1} E T+\left(k_{-1}+k_{2}\right) C, \\
\frac{\mathrm{d} T}{\mathrm{~d} t} & =a T\left(1-b T_{\text {tot }}\right)-k_{1} E T+\left(k_{-1}+k_{3}\right) C, \\
\frac{\mathrm{d} C}{\mathrm{~d} t} & =k_{1} E T-\left(k_{-1}+k_{2}+k_{3}\right) C, \\
\frac{\mathrm{d} E^{*}}{\mathrm{~d} t} & =k_{3} C-d_{2} E^{*}, \\
\frac{\mathrm{d} T^{*}}{\mathrm{~d} t} & =k_{2} C-d_{3} T^{*},
\end{aligned}
$$

where $E, T, C$ are the number of unbound EC, unbound TC, and EC-TC complexes, respectively, located at the site of the tumor, say the spleen, and $E^{*}$ and $T^{*}$ represent the number of inactivated ECs and lethally hit TCs at the tumor site. The total population of unhit TC cells in the spleen is $T_{\text {tot }}=T+C$.

The parameter $s$ is the "normal" (non-enhanced by TC presence) rate of flow of mature EC into the region of TC localization; and $d_{1}, d_{2}$ and $d_{3}$ are positive constants representing the rates of elimination of $E, E^{*}$ and $T^{*}$ cells, respectively, resulting from their destruction or migration from the TC localization area. We assume that the tumor does not metastasize and thus that there is no migration of TC or EC-TC complexes. Such effects could be included in the model by incorporating an additional loss term in equations (1b) and (1c). The maximal growth rate of the TC population is $a$. This parameter incorporates both multiplication and death of TC. The maximal carrying capacity of the biological environment for TC (i.e. the maximum number of cells due, for example, to competition for resources such as oxygen, glucose, etc.) is $b^{-1}$.

The function $F(C, T)$ characterizes the rate at which cytotoxic effector cells accumulate in the region of TC localization due to the presence of the tumor. Both EC multiplication due to stimulation by $\mathrm{TC}$ and enhanced $\mathrm{EC}$ migration 
into this region from surrounding tissues (e.g. nearby lymph nodes) may contribute to the process of EC accumulation. The analysis of Kuznetsov $(1979,1991,1992)$ suggests the following explicit form for this stimulated accumulation of effector cells:

$$
F(C, T)=\frac{f C}{g+T}
$$

where $f$ and $g$ are positive constants. Note that this function depends on $C$, the concentration of EC-TC conjugates. (Below we argue that $C \approx K E T$ ). This functional form is consistent with a model in which one assumes that the accumulation of effector cells is due to signals, such as released cytokines, generated by effector cells in conjugates. Further note that the rate of stimulated accumulation has some maximum value as $T$ gets large. This is consistent with limitations in the rate of transport of effector cells to the tumor. The rate limitation could occur in the circulation, in the rate of exit from the circulation, or in the rate of movement through the tissue to the tumor. Kuznetsov et al. (1993) have recently shown that a function of this form also adequately describes the rate of lymphocyte accumulation into allogeneic tumor spheroids in mice when the concentration of infiltrating macrophages is constant or changes slowly. Also, De Boer and Boerlijst (1993) use a similar function to describe the rate of $T$ cell infection by HIV. In their analysis the rate of infection saturates at high viral burdens.

Equations (1d) and (1e) are "slaves" to equations (1a-c) because the variables $T^{*}$ and $E^{*}$ have no effect on each other or the other variables in the system. In the remainder of this paper we analyse equations $(1 \mathrm{a}-\mathrm{c})$, which dictate the behavior of this system.

The formation and dissociation of cellular conjugates $C$ occurs on a time scale of several tens of minutes to a few hours. A time interval of this order is also observed before the lysis of lethally hit tumor cells (Fishelson and Berke, 1981; Kuznetsov, 1981; Brondz, 1987). However, the multiplication as well as influx of effector cells into the spleen occurs on a much slower time scale, probably tens of hours. This motivates the application of a quasi-steady-state approximation to equation (1c) (i.e. $\mathrm{d} C / \mathrm{d} t \approx 0$ ) which yields the following relation:

$$
C \approx K E T
$$

where $K=k_{1} /\left(k_{2}+k_{3}+k_{-1}\right)$. Experimental observations (Brondz, 1987; Fishelson and Berke, 1981) indicate that EC-TC conjugates usually comprise a small portion of the total number of effector or tumor cells (up to $1-10 \%$ ). This 
motivates the approximation $T_{\text {tot }} \approx T$ which, along with equations (2) and (3), simplifies equations (1a) and ( $1 \mathrm{~b})$ to:

$$
\begin{aligned}
& \frac{\mathrm{d} E}{\mathrm{~d} t}=s+\frac{p E T}{g+T}-m E T-d E, \\
& \frac{\mathrm{d} T}{\mathrm{~d} t}=a T(1-b T)-n E T,
\end{aligned}
$$

where the parameters $p=f K, m=K k_{3}, n=K k_{2}$, and $d=d_{1}$.

3. Parameter Estimates. Experimental data that could serve as a basis for testing mathematical models of the immune response to tumors in vivo are scant. In order to examine if the model, given by equations (4a) and (4b), is adequate we have used the results of experiments on the dynamics of growth of a $\mathrm{BCL}_{1}$ lymphoma in the spleen of chimeric mice (Siu et al., 1986; Uhr et al., 1991), as well as some additional information referred to in the present study.

A number of authors (Strober et al., 1979; Krolick et al., 1979; Weiss et al., 1983; Siu et al., 1986; Uhr et al., 1991) have presented an accurate quantitative description of an experimental model for the interaction in vivo between cytotoxic $\mathrm{EC}$ and $\mathrm{BCL}_{1}$ tumor cells as a function of time. $\mathrm{BCL}_{1}$ was the first $\mathrm{B}$ cell lymphoma described in mice (Slavin and Strober, 1978). It arose spontaneously in an elderly $\mathrm{BALB} / \mathrm{c}$ mouse. The clinical characteristics of $\mathrm{BCL}_{1}$ resemble the prolymphocytic form of chronic lymphocytic leukemia in humans, and hence has been used as a model for that disease (Krolick et al., 1979).

$\mathrm{BCL}_{1}$ has a number of advantages as a model tumor system. First, the cells having been derived from B cells still carry surface immunoglobulin, which can be detected with anti-idiotype reagents. Second, the tumor grows primarily in the spleen and thus studies of tumor growth and tumor-host interactions can focus mainly on this organ. Tumor cells in the spleen can be quantified by idiotypic analysis, and transfer of spleen cells from tumor bearing animals to syngeneic recipients leads to transfer of the tumor. In fact, transfer of a single viable $\mathrm{BCL}_{1}$ cell causes progressive disease in about $50 \%$ of recipients (Krolick et al., 1979).

In normal mice, tumor growth can be detected in the spleen at 3 weeks; growth plateaus by 6 weeks and by 3 months all mice succumb to the tumor (Siu et al., 1986). However, what appears to be a protective immune response can be generated by appropriate experimental manipulation. BALB/c mice can be made chimeric by first having their bone marrow destroyed by lethal irradiation, and then having bone marrow derived from mice of a different $\mathrm{H}-2$ haplotype transferred to restore immune system function. As shown in Fig. 1, 
the $\mathrm{BCL}_{1}$ tumor first grows and then regresses in chimeric mice, and by 12 weeks tumor cells are no longer detectable by anti-idiotypic antibody staining and fluorescence activated cell sorter (FACS) analysis. The initial growth kinetics of the tumor in chimeric animals are very similar to those found in the case of normal BALB/c mice. However, the data in Fig. 1 suggest that in chimeric mice the introduction of large doses of tumor cells invokes a gradually developing strong immune response that leads to the elimination of tumor cells.

The data shown in curve 1 in Fig. 1a describe the growth of the $\mathrm{BCL}_{1}$ lymphoma in non-chimeric animals. We assume that this represents the normal growth of the tumor in the absence of an immune response. In this case the model reduces to:

$$
\frac{\mathrm{d} T}{\mathrm{~d} t}=a T(1-b T)
$$

This equation represents a logistic growth model. Values of $a=0.18 \mathrm{day}^{-1}$ and $b=2.0 \times 10^{-9}$ cells $^{-1}$ give a predicted growth curve that closely approximates the data (see Fig. 1).

The other parameters of the model have been estimated as follows. First

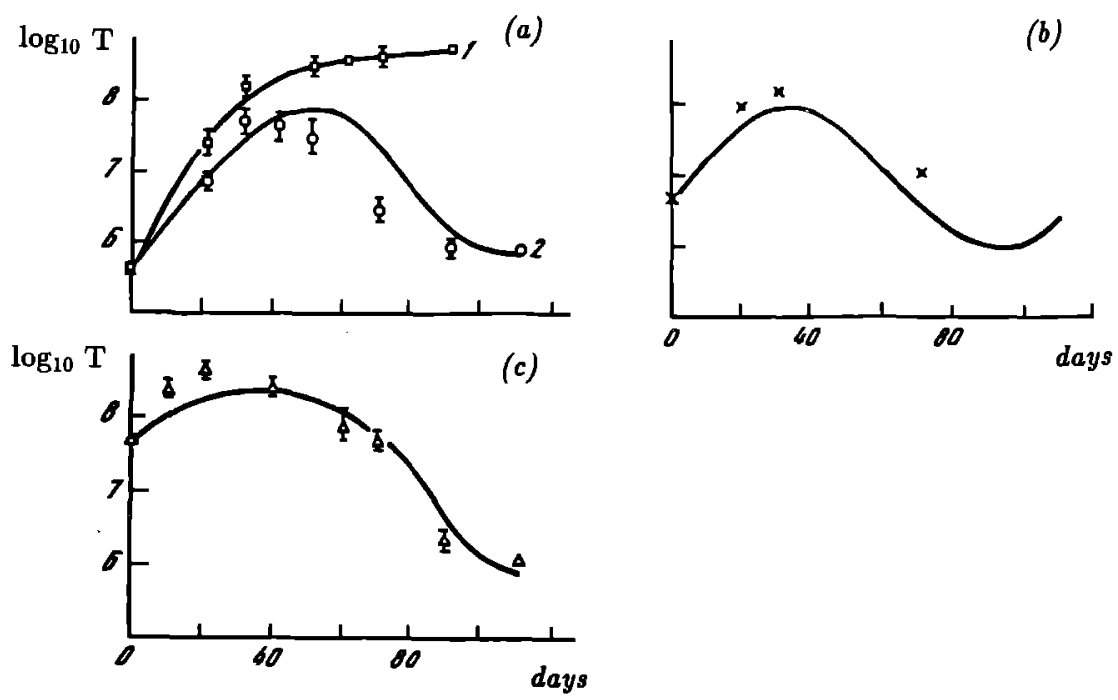

Figure 1. Growth of $\mathrm{BCL}_{1}$ tumor in the spleens of chimeric mice as determined by Siu et al. (1986). Control BALB/c mice received $0.5 \times 10^{6}$ viable $\mathrm{BCL}_{1}$ cells (curve 1 in (a)). Chimeric mice received: $5 \times 10^{5}$ (curve 2 in (a)); $5 \times 10^{6}$ (b); and $5 \times 10^{7}$ (c) viable $\mathrm{BCL}_{1}$ cells. Experimental data $(O, \square, \Delta, x)$ represent the mean of two experiments except in (b) where only one experiment was performed. Theoretical predictions $[-]$ are for $3.2 \times 10^{5}$ initial effector cells at the parameter values presented in the text. 
consider $s$, the normal rate of influx of effector cells into the region of tumor localization. The spleen of a BALB/c mouse contains approximately $10^{8}$ splenocytes. The frequency of CTL precursors reacting to alloantigens forms several tenths of a percent of the total number of lymphocytes (Brondz, 1987). We thus assume that the number of CTL precursors reactive to the tumor, $E_{\mathrm{p}}=3.2 \times 10^{5}$ cells. The lifetime of $\mathrm{T}$ lymphocytes from the spleen and the blood is not known precisely but can be estimated to be approximately 30 days or more (Reynolds et al., 1985; Gray and Leanderson, 1990). We assume that in the absence of a tumor the initial number of CTL, $E(0) \approx E_{\mathrm{p}}$. We further assume that a steady state is established, so that from equation (1a), $s \approx E_{\mathrm{p}} d=1.3 \times 10^{4}$ cells day ${ }^{-1}$. The other parameters, $p, g, m, n$ and $d$, have been estimated from the experimental data given in Fig. 1, using a direct integral method (Yermakova et al., 1982) to obtain a good initial guess for the parameters followed by a non-linear least squares fitting to the data using the Hooke and Jeeves (1961) optimization method (Kuznetsov, 1991). With $a=0.18 \mathrm{day}^{-1}$, $b=2.0 \times 10^{-9}$ cells $^{-1}$, and $s=1.3 \times 10^{4}$ cells day $^{-1}$, we estimate:

$$
\begin{array}{ll}
p=0.1245 \mathrm{day}^{-1}, & g=2.019 \times 10^{7} \text { cells } \\
m=3.422 \times 10^{-10} \mathrm{day}^{-1} \mathrm{cells}^{-1}, & n=1.101 \times 10^{-7} \mathrm{day}^{-1} \mathrm{cells}^{-1} \\
\text { and } & d=0.0412 \mathrm{day}^{-1} .
\end{array}
$$

Parameter estimates are given to four significant places because, as we show below, the initial condition for the experimental data given in Fig. 1c lies close to the separatrix (see Fig. 3) and very small changes in the parameters give noticeable differences in the predicted tumor growth curve. Thus, even though there are not enough data to statistically justify parameter estimates of this degree of precision, we have elected to prescribe parameters to four place accuracy.

The theoretical curves predicted by equations $(4 a, b)$ with these parameter values approximate the experimental values (see Fig. 1). Because all three experimental curves are fit with one set of parameter values no single experiment is fit optimally. Also, it is interesting to note that the predicted regrowth in the tumor population seen in Fig. $1 \mathrm{~b}$ at 100 days has been observed in recent experiments in which $10^{6} \mathrm{BCL}_{1}$ cells were injected into $\mathrm{BALB} / \mathrm{c}$ mice (Uhr et al., 1991).

4. Non-dimensionalization. We non-dimensionalize equations $(4 \mathrm{a}, \mathrm{b})$ by choosing an order-of-magnitude concentration scale for the $\mathrm{E}$ and $\mathrm{T}$ cell populations, $E_{0}$ and $T_{0}$, respectively. As suggested from the experiments 
discussed above: $E_{0}=T_{0}=10^{6}$ cells. Time is scaled relative to the rate of tumor cell deactivation; i.e. $\tau=n T_{0} t$. Then the model can be re-expressed as:

$$
\begin{aligned}
& \frac{\mathrm{d} x}{\mathrm{~d} \tau}=\sigma+\frac{\rho x y}{\eta+y}-\mu x y-\delta x, \\
& \frac{\mathrm{d} y}{\mathrm{~d} \tau}=\alpha y(1-\beta y)-x y,
\end{aligned}
$$

where

$$
\begin{array}{rlrl}
x=\frac{E}{E_{0}}, & y=\frac{T}{T_{0}}, & \sigma=\frac{s}{n E_{0} T_{0}}, & \rho=\frac{p}{n T_{0}}, \\
\eta=\frac{g}{T_{0}}, & \mu=\frac{m}{n}=\frac{k_{3}}{k_{2}}, & \delta=\frac{d}{n T_{0}}, & \alpha=\frac{a}{n T_{0}} \\
\text { and } & \beta=b T_{0} . &
\end{array}
$$

Values for these seven non-dimensional parameters are obtained from the dimensional parameter values estimated in Section 3 to be approximately:

$$
\begin{array}{llll}
\sigma=0.1181, & \rho=1.131, & \eta=20.19, & \mu=0.00311, \\
\delta=0.3743, & \alpha=1.636 & \text { and } & \beta=2.0 \times 10^{-3} .
\end{array}
$$

5. Steady States. Let us consider the steady states of the reduced model described by equations $(6 \mathrm{a}, \mathrm{b})$. Solutions of practical interest will have nonnegative populations $x$ and $y$. We assume that the parameters are also nonnegative. Information regarding both the vector field and the steady states of equations $(6 \mathrm{a}, \mathrm{b})$ can be obtained by examining the nullclines; i.e. the curves along which $\mathrm{d} x / \mathrm{d} t=0$ and $\mathrm{d} y / \mathrm{d} t=0$. There are steady states for the system at the intersections of these nullclines. There is a single nullcline for equation 6(a).

$$
x=\frac{\sigma}{\delta+\mu y-\frac{\rho y}{\eta+y}} \equiv f(y) .
$$

There are two nullclines for equation $6(\mathrm{~b})$. One is $y=0$. The other can be expressed as:

$$
x=\alpha(1-\beta y) \equiv g(y) .
$$

For $\beta>0$, this is simply a straight line with a negative slope. A steady state with 
coordinates $(x, y)=(\sigma / \delta, 0)$ is given by the intersection of $f(y)$ and $y=0$. The stability of this steady state depends upon the relative values of the parameters for the system. Depending upon the relation of $f(y)$ and $g(y)$ there may be from zero to three additional steady states for the system (see Fig. 2). Setting $f(y)$ equal to $g(y)$ yields a third-order polynomial for the $y$ values of these steady states:

$$
C_{3} y^{3}+C_{2} y^{2}+C_{1} y+C_{0}=0
$$

where

$$
\begin{array}{lll}
C_{0}=\eta\left(\frac{\sigma}{\alpha}-\delta\right), & C_{1}=\frac{\sigma}{\alpha}+\rho-\mu \eta-\delta+\delta \eta \beta, \\
C_{2}=-\mu+(\mu \eta+\delta-\rho) \beta & \text { and } & C_{3}=\mu \beta .
\end{array}
$$

In order for equation (8) to have three real roots, it follows from Descartes' rule of signs that there must be three sign changes among the coefficients. Sturm's method (cf. Beaumont and Pierce, 1963) provides more precise conditions for the number of real, distinct roots of equation (8). Table 1 presents a summary of the number of steady states as determined by the signs of the coefficients of equation 8 and the signs of the key quantities appearing in the Sturm sequence of equation (8).

6. Phase Space. For the parameter values estimated in Section 4, there are four steady states predicted by the model. The phase portrait is shown in Fig. 3. The four steady states are indicated and denoted $A, B, C$ and $D$. The variable $x$ is the non-dimensional effector cell population and $y$ the non-dimensional tumor cell population. The steady states labeled $B$ and $D$ are both stable. Steady state $B$ is characterized by a relatively low TC level and we refer to it as the "dormant tumor" steady state. On the other hand, steady state $D$, characterized by a relatively high tumor and low effector cell level, corresponds to relatively "uncontrolled" tumor growth or "tumor escape". The onedimensional stable manifold of steady state $C$ partitions the basins of attraction for each of these attractors. Initial conditions beginning below and the right of this separatrix (e.g. initial conditions (i) and (iii) in Fig. 3) asymptotically approach the dormant tumor steady state $B$. For initial conditions above the separatrix (e.g. initial conditions (ii) and (iv) in Fig. 3), the tumor escapes immune regulation. Thus the model is capable of explaining both tumor dormancy and escape from immunoregulation. In the next sections we delineate the parameter regimes in which these behaviors, as well as "sneaking through" can be expected. 
7. Bifurcation Analysis. Naturally, the parameter values studied above are only estimates. In this section we explore critical parameter values where the qualitative behavior predicted by equations $(6 a, b)$ changes.
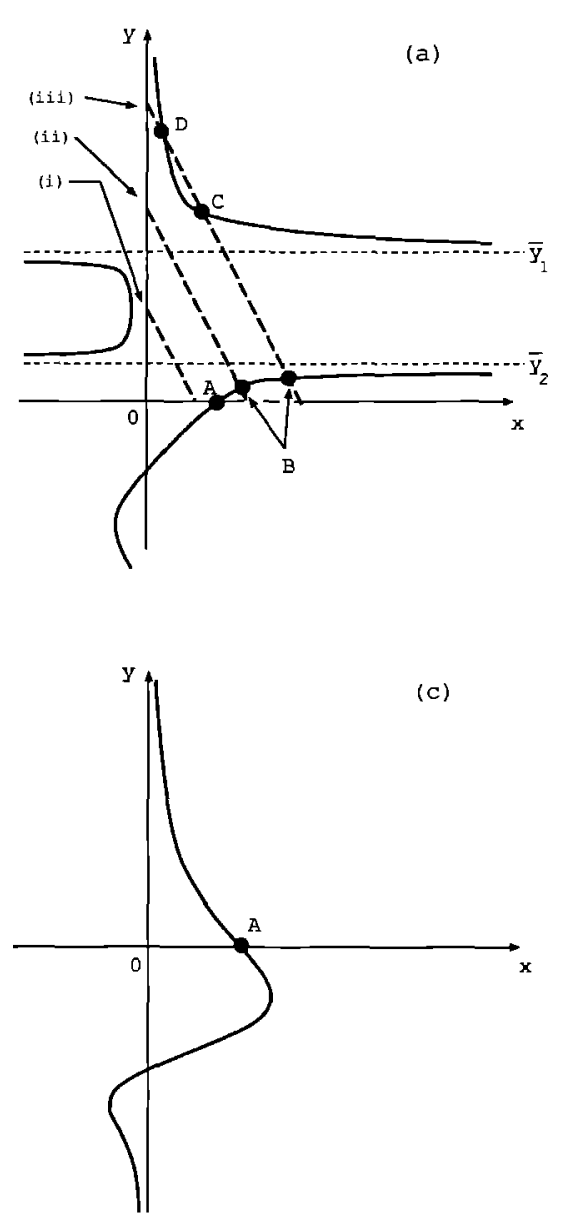
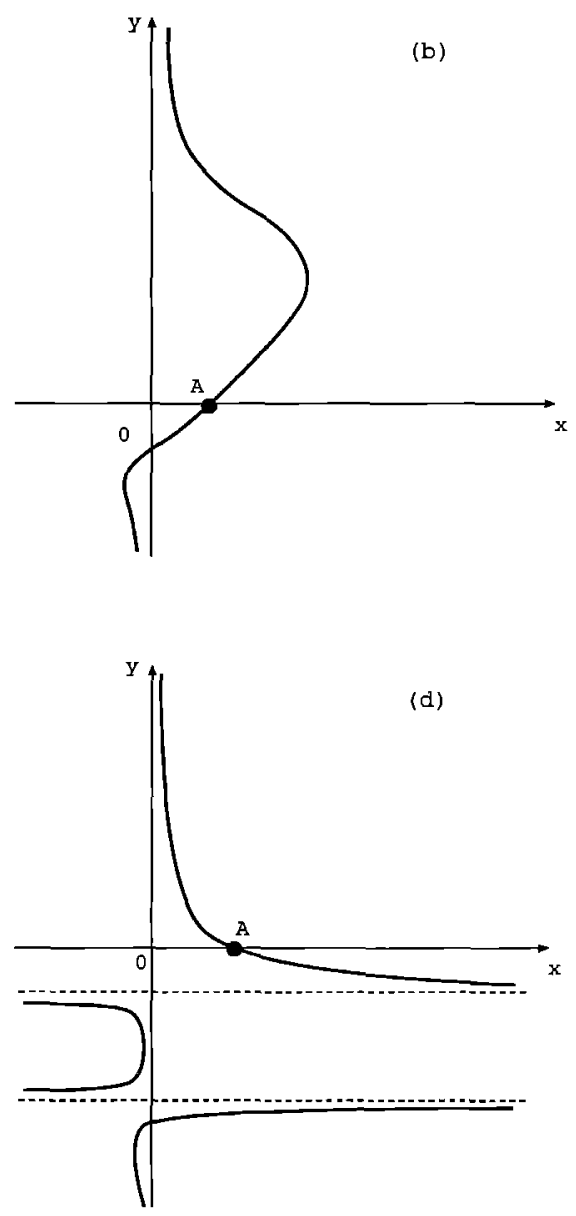

Figure 2. Four qualitatively different forms for $f(y)$ are depicted. These cases are for parameter values such that: (a) $\rho \geqslant(\sqrt{\eta \mu}+\sqrt{\delta})^{2}$; (b) $(\sqrt{\eta \mu}-\sqrt{\delta})^{2}<\rho<(\sqrt{\eta \mu}+$ $\sqrt{\delta})^{2}$ and $\rho>\eta \mu$; (c) $(\sqrt{\eta \mu}+\sqrt{\delta})^{2}<\rho<(\sqrt{\eta \mu}+\sqrt{\delta})^{2}$ and $\rho<\eta \mu$; and (d) $\rho \leqslant(\sqrt{\eta \mu}+\sqrt{\delta})^{2}$, respectively. The horizontal asymptotes $\bar{y}_{1}$ and $\bar{y}_{2}$ for $f(y)$ in (a) and (d) are given by:

$$
\bar{y}_{1,2}=\frac{\rho-\eta \mu-\delta \pm \sqrt{(\rho-\eta \mu-\delta)^{2}-4 \eta \mu \delta}}{2 \mu} .
$$

Three possible orientations for $g(y)$ in relation to $f(y)$ are labeled (i), (ii) and (iii) in (a) which lead to zero, one and three additional positive steady states, respectively. Labeling: $f(y)[-] ; g(y)[---]$; horizontal asymptotes for $f(y)[---]$; and steady states [0]. 
The Dulac-Bendixson criterion (cf. Wiggins, 1990) can be used to show that there are no closed orbits for the system of equations $(4 a, b)$ for positive values of $E$ and $T$. To illustrate, consider the function $M=1 / x y$ and calculate:

$$
\begin{aligned}
L & \equiv \frac{\partial}{\partial x}\left(M \frac{\mathrm{d} x}{\mathrm{~d} t}\right)+\frac{\partial}{\partial y}\left(M \frac{\mathrm{d} y}{\mathrm{~d} t}\right) \\
& =-\left(\frac{\sigma}{x^{2} y}+\frac{\alpha \beta}{x}\right) .
\end{aligned}
$$

Since the parameters are positive, $L<0$ over the domain of interest and the Dulac-Bendixson criterion is satisfied. It follows then that there are no limit cycles or homoclinic connections observed for the system. Similarly, no Hopf bifurcations giving rise to limit cycles occur.

Table 1. The number of positive steady states (SS) as determined by the signs of the coefficients of equation (8) and the signs of the quantities $S=C_{2} C_{1}-9 C_{3} C_{0}$, $T=2 C_{2} S / R-3 C_{3} S^{2} / R^{2}-C_{1}$ and $R=2 C_{2}^{2}-6 C_{3} C_{1}$ from the Sturm sequence. Blank entries correspond to coefficients which may take positive, negative or zero values

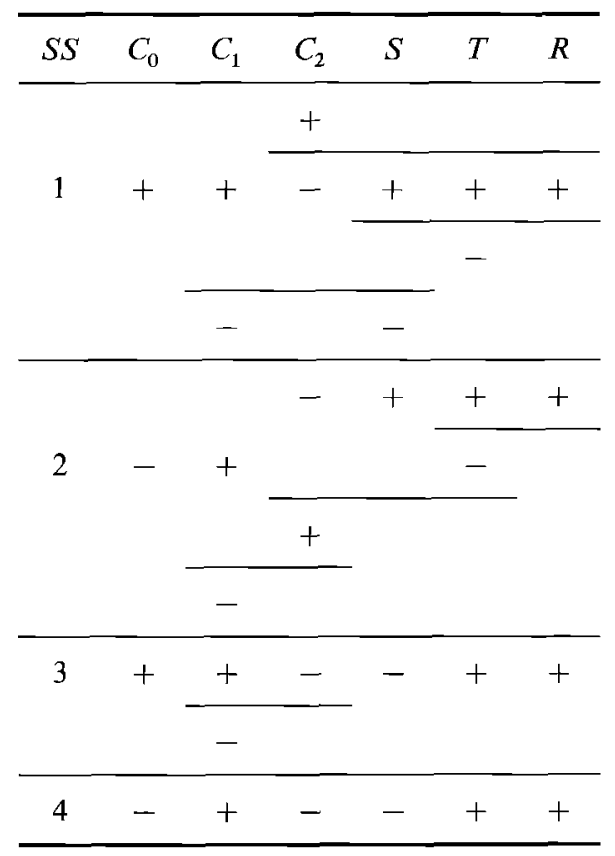



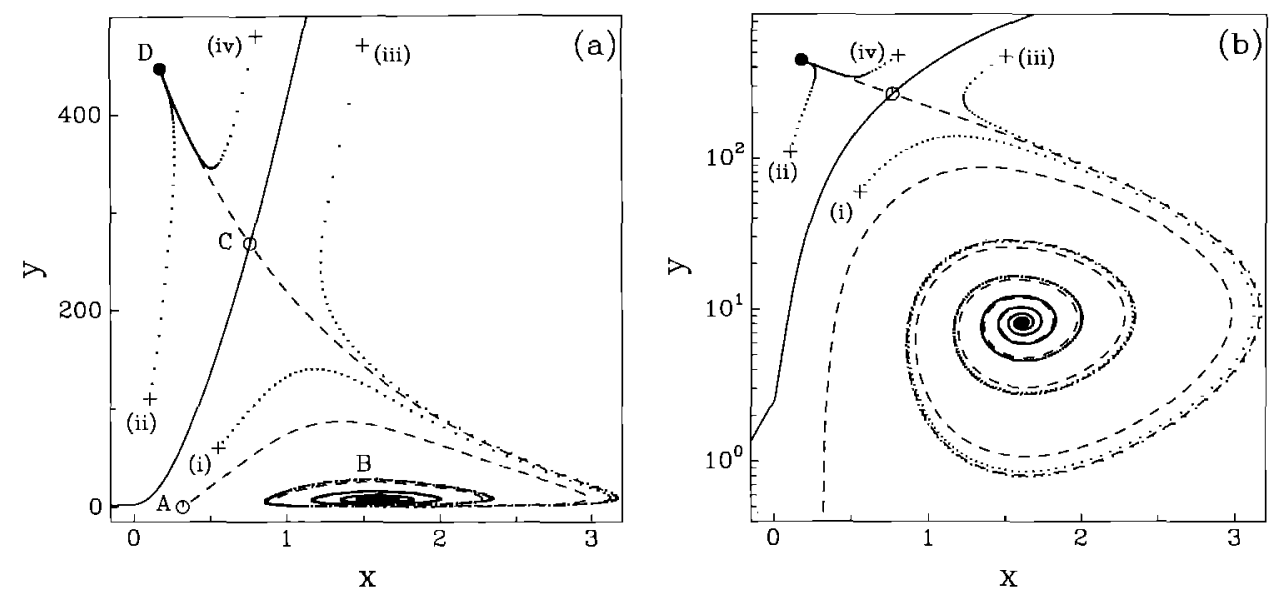

Figure 3. Phase portrait for the model at the dimensionless parameter values presented in Section 4. Here $x$ is the non-dimensional effector cell population level and $y$ is the non-dimensional tumor cell population. Portrait (a) is redrawn with a logarithmic ordinate in portrait (b). Labeling: stable steady states [0], saddle steady states $[O]$, one-dimensional stable manifold of steady state $C[-]$, onedimensional unstable manifolds of steady states $A$ and $C[---]$, initial conditions for transients [+] (denoted (i) (iv), and the evolution of transients in time increments of 1 day $[\cdots]$.

To better understand the behavior predicted by equations $(6 a, b)$, we have mapped out qualitatively different regions of behavior as a function of the parameters $\delta$, the effector cell "death rate", and $\sigma$, the baseline effector cell "source rate", at the fixed values of the other parameters. These results are presented in Fig. 4a. Curves in this diagram represent codimension-one bifurcations which partition parameter regions of qualitatively different dynamic behavior. Representative phase portraits for regions $1-5$ are depicted in Figs 5a-e, respectively. The transcritical bifurcation curve and the saddlenode bifurcation curve involve different pairs of steady states. They appear to intersect in Fig. 4 only because the solution $\times$ parameter $\left(\mathbb{R}^{2} \times \mathbb{R}^{2}\right)$ space is being projected onto a plane.

The loci of saddle-node and transcritical bifurcations presented in Fig. 4 are termed "local" bifurcation curves because they are characterized by a qualitative change in the linearized stability evaluated at the steady state. A qualitatively different codimension-one bifurcation which is not observable from the local linearization forms the transition between regions 3 and 5 . This "global" bifurcation is a heteroclinic connection where one side (the lower-left portion) of the one-dimensional stable manifold of steady state $C$ and the nonnegative portion of the one-dimensional unstable manifold of steady state $A$ coincide. The effect of this bifurcation is to radically change the territory claimed by competing attractors and is illustrated schematically in Fig. 6. This 
bifurcation has biological significance (see below) and has been previously called a "vital barrier" (Kuznetsov, 1983, 1988).

Parameter estimates place our system in region 3 (marked $\bullet$ in Fig. 4). In this region, all trajectories beginning with high numbers of effector cells, $x$, and low numbers of tumor cells, $y$ (corresponding to the unshaded region in Fig. 6b) asymptote to the dormant tumor state.

Our parameter estimates place the system close to the global bifurcation boundary between regions 3 and 5 . Thus, with a slight change in parameters the system can be in region 5. Interestingly, many experimentally observed

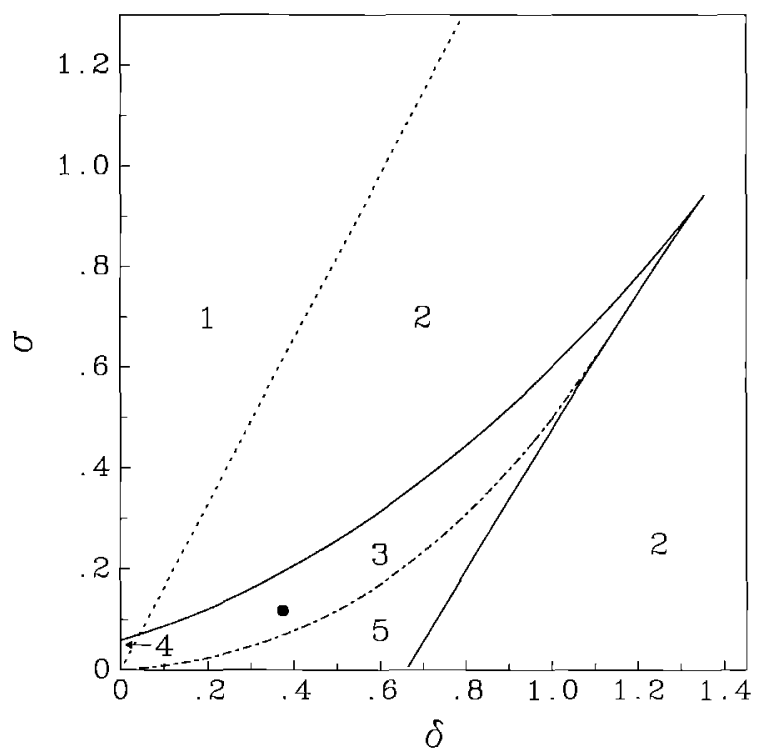

\begin{tabular}{ll}
\hline \multicolumn{1}{c}{ Transition } & \multicolumn{1}{c}{ Brief description } \\
\hline $1-2,4-3$ & Transcritical bifurcation involving steady states $A$ and $B$ \\
$1-4,2-3$ & Saddle-node bifurcation involving steady states $C$ and $D$ \\
$2-5$ & Saddle-node bifurcation involving steady states $C$ and $B$ \\
$3-5$ & $\begin{array}{l}\text { Heteroclinic connection involving steady states } A \text { and } C \\
\end{array}$ \\
& Associated with a significant change in the basins of attraction \\
& for the attractors.
\end{tabular}

Figure 4. The two-dimensional transition structure (including both local and global bifurcations) as a function of $\delta$, the effector cell death rate, and $\sigma$, the effector cell source rate, at the fixed values of the other dimensionless parameters presented in Section 4. The two-parameter diagram contains five distinct regions, labeled 1-5. The table summarizes the transitions between each of these regions. Figure 5 shows a representative phase portrait for each of these regions. Labeling of bifurcation curves: saddle-node [-]; transcritical bifurcation [---]; and heteroclinic connection [------]. The estimated parameter values for our model; 
phenomena are characteristic of the behavior predicted in region 5 . In region 5 transients beginning with high effector cell levels, i.e. high $x$ levels (e.g. initial condition (ii) in portrait 5e) quickly approach an apparent dormancy where the tumor presence is reduced but not eliminated. However, the tumor level persists over time as the effector cell level gradually drops. Eventually the tumor escapes and the system approaches steady state $D$. This is an illustration of the "sneaking through" phenomenon. An external stimulation to an immune system in region 5, which may seem intuitively to aid the immune response (immunostimulation, e.g. perturbing from initial condition (i) to (ii) in Fig. 5e) can actually be detrimental. In addition, sufficiently small tumors (below the separatrix in portrait 5e) are predicted to eventually escape immune regulation.
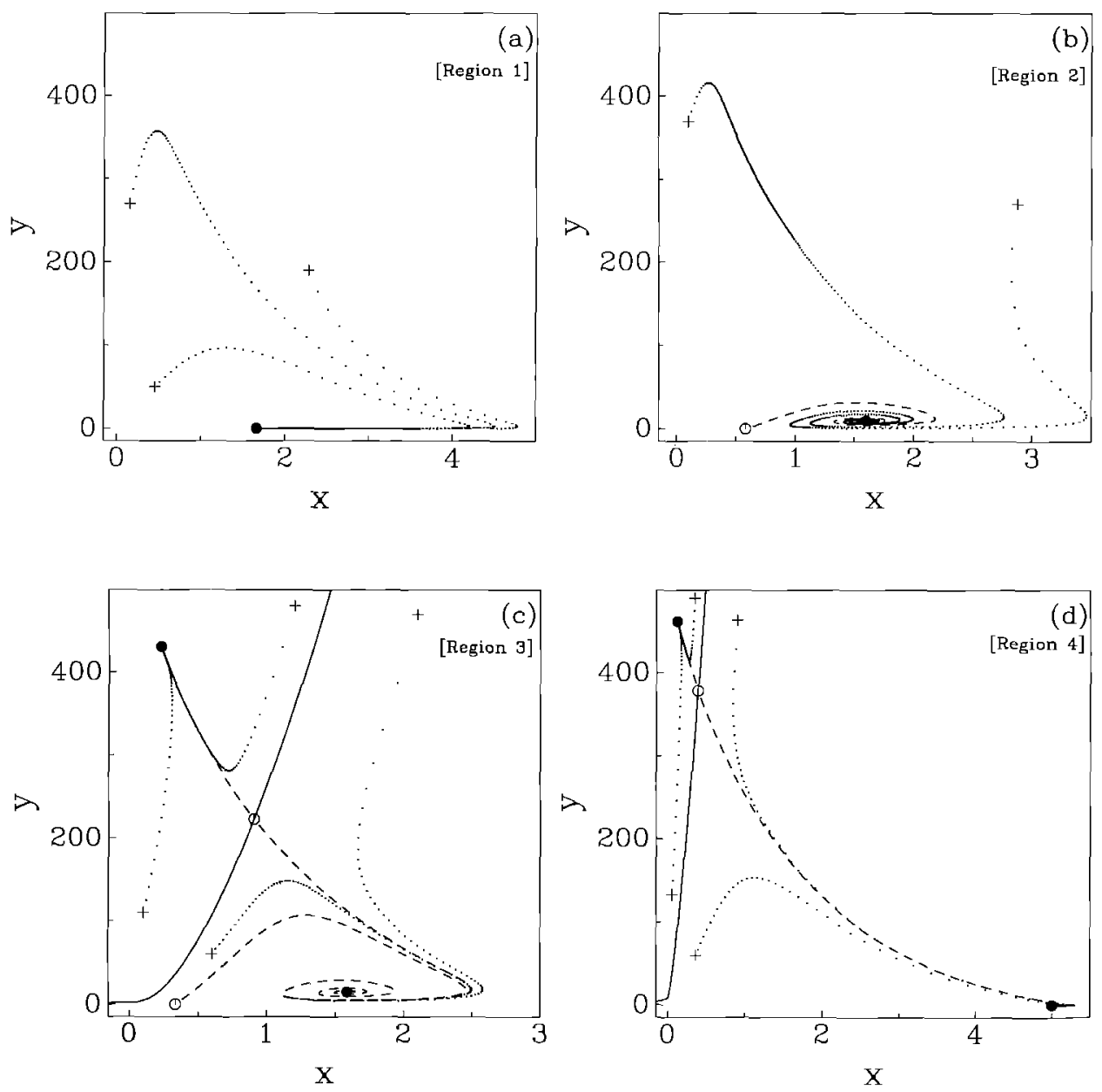

Figures 5a-d. 

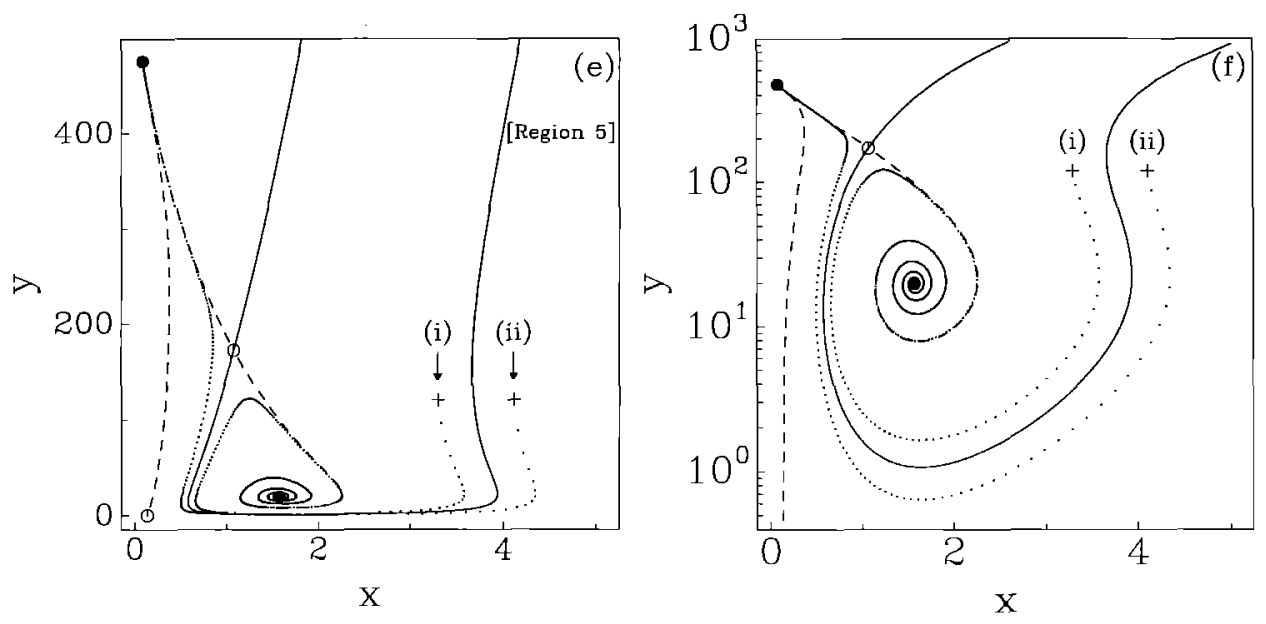

Figures $5 \mathrm{e}$ and $\mathrm{f}$.

Figure 5. Representative phase portraits for each of the regions in Fig. 4. Recall $x$ is the non-dimensional effector cell level and $y$ is the non-dimensional tumor cell level. Portraits (a)-(e) (which represent regions 1-5, respectively, are at $(\delta, \sigma)$ values of $(0.1908,0.318),(0.545,0.318),(0.545,0.182),(0.009,0.045)$ and $(0.545,0.073)$, respectively. Portrait (e) is redrawn with a logarithmic ordinate in portrait (f). Labeling: stable steady states [O], steady states with saddle stability [O], onedimensional stable manifolds of saddles $[-]$, one-dimensional unstable manifolds of saddles $[--]$, initial conditions for transients $[+]$, and the evolution of transients in time increments of 1 day $[\cdots]$.

8. Discussion. Given the limited data of Fig. 1, the numerical estimates of the model parameters for the response to $\mathrm{BCL}_{1}$ cells should only be regarded as preliminary. However, we have some confidence in our estimates of the parameters $\alpha$ and $\beta$ characterizing tumor growth in the absence of an immune response, since the logistic model is biologically reasonable and the two parameters that need to be estimated from the data determine the slope and asymptote of the tumor growth curve. As shown in Fig. 1, the logistic curve with our parameter estimates fits the data well over a wide range of initial
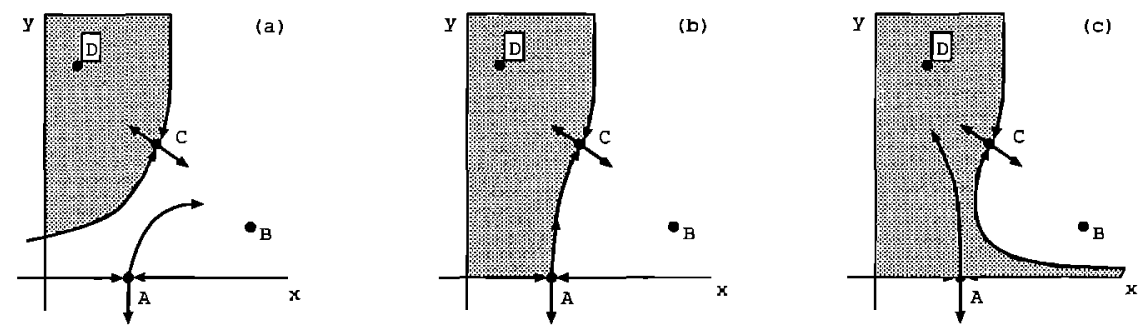

Figure 6. Representative phase portraits: (a) before, (b) during, and (c) after; the heteroclinic connection between steady states $A$ and $C$. Portions of the basins of attraction for attractors $B$ and $D$ are the white and shaded regions, respectively. 
tumor cell concentrations. If independent measurements of other parameters were available, they could help guide interpretation of the processes of interaction between the host immune system and a growing tumor. However, most of the relevant parameters have not been measured in vivo and thus we have used bifurcation theory to study the behavior of the model as parameters are varied.

Using our parameter estimates, we can make a number of biologically interesting predictions about the interactions of the immune system with a growth tumor. In Fig. 3 a phase portrait of the system is presented at the parameter values estimated in Section 4 . Transients in the vicinity of steady state $B$ exhibit decaying oscillations. A time series for an initial condition in this neighborhood is presented in Fig. 7. The result is of interest from the standpoint of predicting the behavior of the tumor over greater time-scales than those measured in Siu et al. (1986). Cyclic fluctuations in the number of leukocytes has been found in a number of cases in the development of chronic human myeloid leukemia (Menta and Agarwal, 1980) and chronic bovine lymphoid leukemia (Kukain et al., 1982). This pattern of spontaneous relapse and remission is reminiscent of observations in non-Hodgkin's lymphona (Krikorian et al., 1980). It is also interesting that the predicted time scale of oscillations in the model, 3 or 4 months, is in rough agreement with the time for recurrent clinical manifestations of certain human leukemias. In addition,

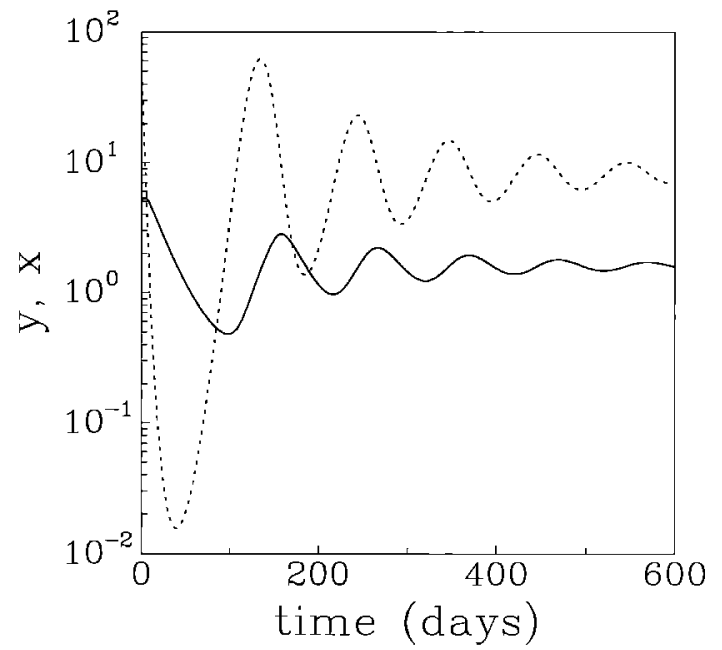

Figure 7. The time course of $\mathrm{BCL}_{1}$ leukemia $y$ [---] and effector $x$ cell levels [-] in chimeric mice, illustrating a decaying oscillation to the dormant tumor state. The dimensionless initial number of leukemia cells is $y=50$ and the dimensionless initial number of effector cells is $x=5$. Dimensionless parameter values are those presented in Section 4. 
recurrent patterns of tumor remission and regrowth have been seen in the $\mathrm{BCL}_{1}$ system for mice challenged with $10^{6} \mathrm{BCL}_{1}$ cells (Uhr et al., 1991).

Another prediction of the model follows from Fig. 3. In a mouse with a typical effector cell concentration of $0.5 \times 10^{6}(x=0.5)$, when more than approximately $1.5 \times 10^{8}$ tumor cells $(y=150)$ are injected into the animal, our model predicts that the resultant tumor growth should not be controlled by the immune system. However, if fewer tumor cells are injected, then tumor growth is predicted to be controlled. This prediction of an "immunological barrier" value can be easily checked experimentally.

In reports by Strober et al. (1979) and Weiss et al. (1983) data are presented that suggest that immunological mechanisms induce the formation of a dormant state of $\mathrm{BCL}_{1}$ tumor in chimeric mice. In this state potentially lethal tumor cells persist in the animal with little or no increase in their population. Our model predicts the existence of a dormant state (steady state $B$ in Fig. 3).

In certain animals it is not possible to detect leukemia cells several months after the administration $\mathrm{BCL}_{1}$ cells, even if $10^{6}$ splenocytes from these regressor mice are transplanted into non-chimeric BALB/c mice (Weiss et al., 1983). In this regard it is of interest to note that total regression may be obtained with the model given by equations $(6 a, b)$ only when there is a sufficiently pronounced change in the parameters from our estimated values, for example, a six-fold increase in the $\sigma / \delta$ ratio. In this case the rate of growth of the tumor cell population will be less than their death rate $(\alpha>\sigma / \delta)$ and solutions emerge in which the tumor cell population $y$ approaches zero.

As a body ages the probability of transformed cells arising is thought to increase and the reliability of immune surveillance mechanisms probably decreases. Within the framework of our mathematical model such changes may be interpreted as: (1) an increase in the initial number of tumor cells at the time the immune system encounters the tumor, and a reduction of the threshold for its macroscopic growth, or (2) the reduction of the area in parameter space that corresponds to the total elimination of a primary tumor (i.e. where solutions $y$ tend to 0 ) and the growth of areas in which either the system is in dynamic balance or the tumor is not controlled by the system. In either of these variants of the system even a single tumor cell may develop into a small "dormant" tumor. The number of such tumors is thus expected to increase with the organism's age until some stochastic event causes a change in the tumor growth rate, access to lymphocytes, or some other parameter changes such that a bifurcation border is crossed and the uncontrolled tumor growth begins. In this regard it is interesting to note that the experiments of Yefenof et al. (1993) suggest that a mutation causes a change in dormant $\mathrm{BCL}_{1}$ cells that allows them to escape the dormant state.

It follows from our bifurcation analysis that at certain parameter values solutions are possible that can be interpreted as tumor sneaking through and 

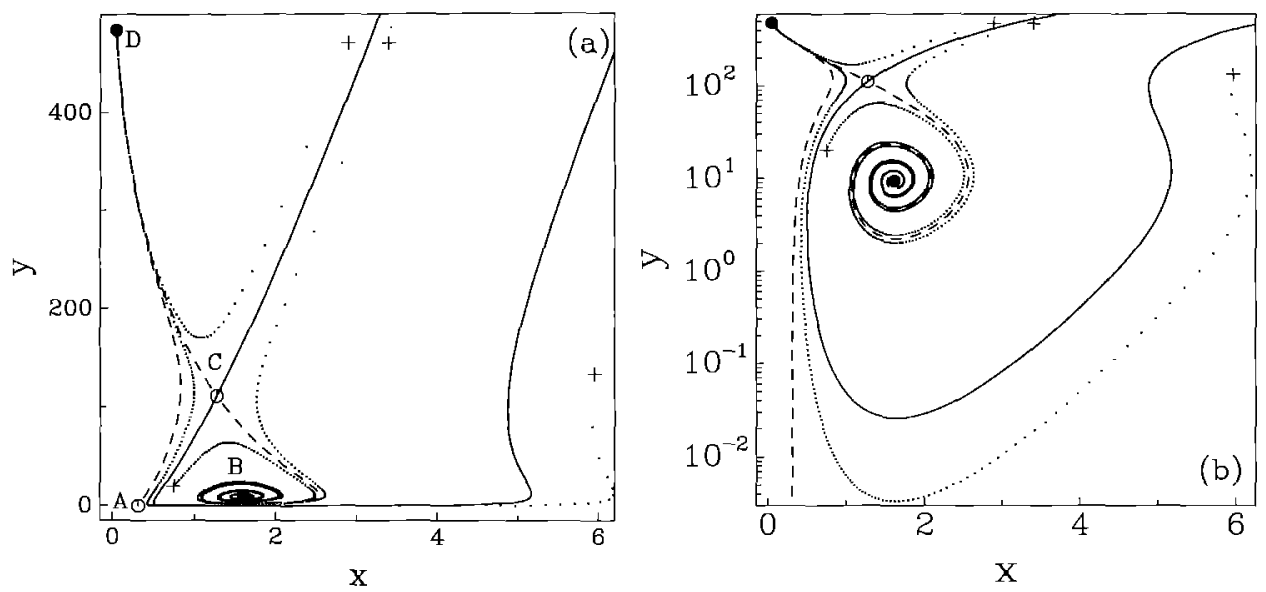

Figure 8. A typical phase portrait for $\mu$ slightly greater than 0.005014 . For this portrait $\mu=0.0055$ and the other dimensionless parameter values are those presented in Section 4. Portrait (a) is redrawn with a logarithmic ordinate in portrait (b). Labeling: stable steady states [O], saddle steady states [O], onedimensional stable manifold of steady state $C[-]$, one-dimensional unstable manifolds of steady states $A$ and $C[---]$, initial conditions for transients $[+]$, and the evolution of transients in time increments of 1 day $[\cdots]$.

immunostimulatory effects. Sneaking through refers to a phenomena in which low doses of tumor cells can escape immune defenses and grow into a large tumor, whereas larger doses of tumor cells are eliminated. Numerical continuation has shown that the parameter values we have estimated for the system lie close to what has been called a "vital barrier" transition (Kuznetsov, $1983,1988)$. On one side of the barrier sneaking through is possible, whereas on the other side it is not. The possible behaviors of our model are very sensitive to the parameter $\mu=k_{3} / k_{2}$. For example, as $\mu$ increases the one-dimensional manifolds of steady states $C$ and $A$ cross in a heteroclinic connection at $\mu=0.005014$. After the heteroclinic connection, the phase portrait of the system (e.g. for $\mu=0.0055$ in Fig. 8) is qualitatively similar to portrait 5e and exhibits the phenomena of sneaking through and immunostimulation leading to tumor escape. On the other hand, the possibility of tumor escape is lost as $\mu$ decreases since steady states $C$ and $D$ collide in a saddle-node bifurcation at $\mu=0.002633$. After the saddle-node, the phase portrait of the system is qualitatively similar to portrait 5 b (e.g. for $\mu=0.0021$ in Fig. 9).

It is clear that small fluctuations of parameter values take place in vivo. Neither the effector cell nor tumor cell populations are homogeneous. Differing subpopulations will have different parameter values characterizing their behavior. Because of the sensitivity of the model's behavior to parameter values, we predict the ultimate instability of the dormant state for $\mathrm{BCL}_{1}$. Moreover, we predict that the times of clinical manifestation of tumor growth 

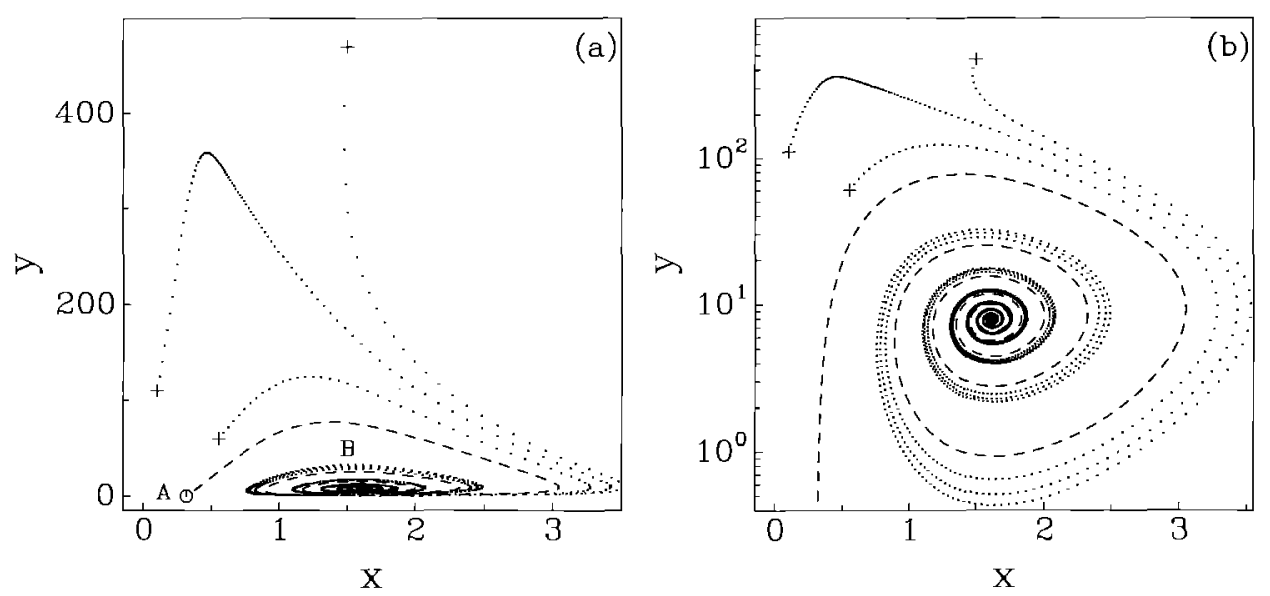

Figure 9. A typical phase portrait for $\mu$ slightly less than 0.002633 . For this portrait $\mu=0.0021$ and the other dimensionless parameter values are those presented in Section 4. Labeling: stable steady states [0], saddle steady states [0], onedimensional unstable manifold of steady state $A[---]$, initial conditions for transients [+], and the evolution of transients in time increments of 1 day $[\cdots]$.

should be stochastic. From our numerical experiments, shown in Fig. 6, we see that small changes of initial conditions in the sneaking through region lead to very large differences in the time period needed for the appearance of large tumors. These results correspond to observations on the emergence from the dormant state for BLC 1 lymphoma (Krolick et al., 1979; Strober et al., 1979; Siu et al., 1986; Uhr et al., 1991) as well as for other experimental models of the tumor dormant state (Weinhold et al., 1979a,b; Uyttenhove et al., 1983; Hiernaux et al., 1986).

The above observations make it clear that $\mu$ is a critical parameter in the model. Our estimate, $\mu=0.003$, is sufficiently small to make one wonder if in the biological system $\mu$ is not actually zero. Recall that $\mu=k_{3} / k_{2}$, and hence $\mu$ small or zero implies that $k_{3} \simeq 0$, where $k_{3}$ is the rate at which effector cells are inactivated due to interaction with the tumor. Thus with small $\mu$, our model predicts that $\mathrm{BCL}_{1}$ tumors do not efficiently inactivate effector cells. The argument given above, however, shows that the phenomena of sneaking through and immunostimulation of tumor growth depend on $\mu$ being above 0.002633 . Thus, according to our model, a rather small rate of effector cell inactivation is needed to generate the phenomena seen in vivo. Our model thus suggests that it would be worthwhile to accurately monitor the ability of different tumors to inactivate effector cells and correlate that ability with observation of sneaking through and immunostimulation.

The mathematical model presented here shows that the phenomena of sneaking through, immunostimulation and the presence of dormant tumors 
may be related effects. We have used the term effector cells in our presentation. These cells may be CTL, NK cells, macrophages, the effector cells of antibody dependent cellular cytotoxicity reactions, or any other cell involved in immune surveillance against tumors. Thus, we believe the detailed mechanisms leading to the phenomena of sneaking through, immunostimulation and dormant tumors may be rather diverse. For particular tumors it is still necessary to determine the prevailing effector mechanism(s) leading to the stabilization and regression of a tumor growth in vivo and to obtain detailed experimental data regarding the kinetics of the relevant immune processes. Nevertheless, we feel that the model that we have presented is generic and relies on properties that any effector cell population should exhibit. Thus, the transitions in the behavior of the system as parameters are varied should have a universal character, that we expect will be seen in a variety of biological situations. It is for this reason that bifurcation diagrams, such as the one given in Fig. 4, may be quite helpful in interpreting experimental data.

Although our model and its analysis were aimed at understanding tumor growth, it may have broader applicability. De Boer and Boerlijst (1993) have introduced equations (6a) and (6b), with $\beta=0$, as a model for human immunodeficiency virus (HIV) infection in AIDS. In their model $x$ represents the concentration of HIV specific $\mathrm{CD} 4^{+}$helper $\mathrm{T}$ cells that are involved in the immune response against HIV. These cells are assumed to be created at rate $\sigma$ and die with a per capita death rate $\delta$. The virus, HIV, at concentration $y$, stimulates $\mathrm{T}$ cell growth to a maximum proliferation rate $\rho$ in a density dependent manner with $\eta$ being the saturation constant of the process. HIV also kills $\mathrm{T}$ cells at rate $\mu x y$. Equation (6b) describes the kinetics of viral growth and elimination. De Boer and Boerlijst assume virus grows at rate $\alpha y$ and is eliminated by an immune response at rate $c x y$. In equation (6b), due to our scaling $c=1$. Since HIV kills T cells there is no question that $\mu$ in equation (6a) should be non-zero in this application. Kuznetsov $(1983,1992)$ has analysed the possible bifurcations with $\beta=0$. One could also envision circumstances in which $\beta$ were positive and the virus grew logistically, in which case the model of De Boer and Boerlijst would become identical to the model presented here. This raises the possibility of sneaking through in AIDS by the mechanism elucidated in this paper.

9. Conclusions. A quantitative model has been proposed for the interaction between effector cells and cells in a growing tumor. The model adequately describes the kinetics of growth and regression of a $\mathrm{BCL}_{1}$ lymphoma in the spleen of chimeric mice over a wide initial tumor cell concentration range. Hypothesizing that cytotoxic effector cells are responsible for the antitumor reactivity seen in the experimental model, we find that the model can account for many phenomena observed in vivo. Local and global bifurcations for 
realistic values of the parameters were calculated, and show that there may be a connection between the phenomena of immunostimulation of tumor growth, "sneaking through" of tumor, and formation of the tumor "dormant" state.

According to our model, the limited growth of even the high initial $\mathrm{BCL}_{1}$ tumor cell concentrations in the chimeric animals is associated with a high rate of accumulation of specific, highly-active, cytotoxic effector cells in the spleen and with the absence of suppression of the cytotoxic activity of these cells by the tumor. A threshold number of tumor cells, equivalent to $8 \times 10^{7}$ cells for the analysed experimental model, is predicted above which immunologically uncontrollable tumor growth should be found and below which attenuation of the disease with periodic exacerbations and clinical manifestation every 3-4 months occur.

One can speculate that even in the presence of cell mediated immune responses, which in our model do not totally eliminate even highly immunogenic tumors, a multitude of dormant tumors could accumulate in body tissues with increasing age. Thus, according to our model it would not be surprising to observe an increased frequency of tumors as an animal ages.

The model may also be applicable to other processes in biology such as the infection of $\mathrm{T}$ cells by HIV.

One of the authors (VK) acknowledges the assistance of Yu. I. Skal'ko with some of the calculations, and wishes to thank the Santa Fe Institute for their hospitality. We also thank Dr Steven Skates for valuable comments about a preliminary draft of the manuscript. This work was performed under the auspices of the U.S. Department of Energy. It was supported in part by NIH Grant AI28433, by the Center for Nonlinear Studies at Los Alamos, and by the Santa Fe Institute through their Theoretical Immunology Program.

\section{REFERENCES}

Abrahms, S. I. and Z. Brahmi. 1988. Mechanism of K562-induced human natural killer cell inactivation using highly enriched effector cells isolated via a new single-step sheep erythrocyte rossette assay. Ann. Inst. Pasteur, Immunol. 139, 361-381.

Albert, A., M. Freedman and A. S. Perelson. 1980. Tumors and the immune system: The effects of a tumor growth modulator. Math. Biosciences 50, 25-58.

Alsabti, A. 1978. Tumor dormancy: A review. Tumor Res. 13, 1-13.

Beaumont, R. A. and R. S. Pierce. 1963. The Algebraic Foundations of Mathematics. Reading, MA: Addison-Wesley.

Brondz, B. D. 1987. T Lymphocytes and Their Receptors in Immunological Recognition (in Russian). Moscow: Nauka.

Callewaert, D. M., P. Meyers, J. Hiernaux and G. Radcliff. 1988. Kinetics of cellular cytotoxicity mediated by cloned cytotoxic T lymphocytes. Immunobiol. 178, 203-214.

Chen, L., Y. Suzuki, C.-M. Liu and E. F. Wheelock. 1990. Maintenance and cure of the L5178Y murine tumor dormant state by interleukin 2: Dependence of interleukin 2 on induced inteferon-g and on tumor necrosis factor for its antitumor effects. Cancer Res. 50, 1368-1374. 
Colmeraver, M. E., I. A. Loziol and V. H. Pilch. 1980. Enhancement of metastasis development by BCG immunotherapy. J. Surg. Oncology 15, 235-241.

De Boer, R. J. and M. C. Boerlijst. 1993. Diversity and virulence thresholds in AIDS (submitted).

De Boer, R. J. and P. Hogeweg. 1985. Tumor escape from immune elimination: Simplified precursor bound cytotoxicity models. $J$. theor. Biol. 113, 719-736.

De Boer, R. J. and P. Hogeweg. 1986. Interactions between macrophages and T-lymphocytes: Tumor sneaking through intrinsic to helper T cell dynamics. J. theor. Biol. 120, 331-354.

Deichman, G. I. 1979. Current concepts on the immunological interaction between the tumor and the body. In Tumor Growth as Problem of Development Biology, pp. 208-223. Moscow: Nauka.

Deichman, G. I., T. E. Klyuchareva, L. M. Kashkina and V. A. Matveyeva. 1979. Reproducibility and relation to specific and nonspecific antitumor resistance of the "sneaking through" phenomenon. Int. J. Cancer 23, 571-584.

DeLisi, C. and A. Rescigno. 1977. Immune surveillance and neoplasia-1. A minimal mathematical model. Bull. math. Biol. 39, 201-221.

Dozmorov, I. M. and V. A. Kuznetsov. 1988. The role of cellular ratios in the maintenance of organism immune homeostasis. In Problems and Perspectives of Modern Immunology: Methodological Analysis (in Russian), R. V. Petrov and V. P. Lozovoy (Eds), pp. 43-66. Novosibirsk: Nauka.

Emanuel, N. M. 1981. Chemical and biological kinetics. Russian Chem. Rev. 50, 901-947.

Fidler, I. J. 1973. In vitro studies of cellular-mediated immunostimulation of tumor growth. $J$. Natl Cancer Inst. 50, 1307-1312.

Fishelson, Z. and G. Berke. 1981. Tumor cell destruction by cytotoxic T lymphocytes: The basis of reduced antitumor cell activity in syngeneic hosts. $J$. Immunol. 125, 2048-2052.

Gatenby, P. A., A. Basten and P. Creswick. 1981. "Sneaking through": A T-cell-dependent phenomenon. Br. J. Cancer 44, 753-756.

Gray D. and T. Leanderson. 1990. Expansion, selection and maintenance of memory B-cell clones. Current Topics Microbiol. Immunol. 159, 1-17.

Greenberg, P. D. 1991. Adoptive T cell therapy of tumors: Mechanisms operative in the recognition and elimination of tumor cells. Adv. Immunol. 49, 281-355.

Grossman, Z. and G. Berke. 1980. Tumor escape from immune elimination. $J$. theor. Biol. 83, $267-296$.

Hellström, K. E. and I. Hellström. 1969. Cellular immunity against tumor antigens. Adv. Cancer Res. 12, 167-223.

Herberman, R. B. 1974. Cell-mediated immunity to tumor cells. Adv. Cancer Res. 19, 207-263.

Hiernaux, J. R., R. Lefever, C. Uyttenhove and T. Boon. 1986. Tumor dormancy as a result of simple competition between tumor cells and cytolytic effector cells. In Paradoxes in Immunology, G. W. Hoffman, J. G. Levy and G. T. Nepom (Eds), pp. 95-109. Florida: CRC Press.

Hooke, R. and T. A. Jeeves. Direct search solution of numerical and statistical problems. $J$. Assoc. Comput. Machin. 8, 212-229.

Jeejeebhoy, H. F. 1977. Stimulation of tumor growth by the immune response. Int. J. Cancer 13, 665-678.

Krikorian, J. G., C. S. Portlock, D. P. Cooney and S. A. Rosenberg. 1980. Spontaneous regression of non-Hodgkin's lymphoma: A report of nine cases. Cancer 46, 2093-2099.

Krolick, K. A., P. C. Isakson, I. W. Uhr and E. S. Vitetta. 1979. BCL 1 , a murine model for chronic lymphocytic leukemia: Use of the surface immunoglobulin idiotype for the detection and treatment of tumor. J. Immunol. Rev. 48, 81-106.

Kukain, R. A., L. I. Nagayeva, V. P. Lozha, S. Ya Laganovsky, S. V. Chapenko, O. I. Bratsslavskaya, V. P. Ose and G. V. Kudeleva. 1982. Bovine Leukemia Virus (in Russian). Riga: Zinatne.

Kuznetsov, V. A. 1979. The dynamics of cellular immunological antitumor reactions. I. Synthesis of a multi-level model. In Mathematical Methods of Systems Theory (in Russian), Vol. 1, pp. 57-71. 
Kuznetsov, V. A. 1981. A model for cytotoxic cellular immune process and its experimental application (in Russian). In Applied Problems in the Theory of Dynamic Systems, Gorky, Vol. 4, pp. 14 43. Manuscript submitted to the All-Union Institute of Science and Technology Information, 25 December 1981, No. 5851.

Kuznetsov, V.A. 1983. Bifurcations in a model of the two-level reactivity of an immune system to antigens of a developing neoplasm. In Dynamics of Biological Populations, Gorky (in Russian), pp. 52-64. Gor'ki State University.

Kuznetsov, V.A. 1984. Analysis of population dynamics of cells that exhibit natural resistance to tumors. Soviet Immunol. (Immunologiya) 3, 58-68.

Kuznetsov, V. A. 1987. Mathematical modelling of the processes of dormant tumors formation and immunostimulation of their growth (in Russian). Cybernetics 4, 96-102.

Kuznetsov, V. A. 1988. Nonlinear effects of the dynamics of antitumor cellular immune system (preprint; in Russian). Moscow: Institute of Chemical Physics, Academy of Sciences, USSR.

Kuznetsov, V. A. 1991. A mathematical model for the interaction between cytotoxic lymphocytes and tumour cells. Analysis of the growth, stabilization and regression of the B cell lymphoma in mice chimeric with respect to the major histocompatibility complex. Biomed. Sci. 2, 465-476.

Kuznetsov, V. A. 1992. Dynamics of Immune Processes During Tumor Growth (in Russian). Moscow: Nauka.

Kuznetsov, V. A., A. V. Inshina and Z. G. Kadagidze. 1988. Computer-aided determination of the number of active natural killers, their avidity and the rate of recycling in a lytic cycle. Soviet Immunology (Immunologiya) 5, 25-30.

Kuznetsov, V. A. and M. V. Volkenshtein. 1978. Mathematical model of cellular immune response to tumor growth (in Russian). In The Reports at the Third All-Union Conference on Biology and Medical Cybernetics (Sukhumi), pp. 58-61. Moscow: USSR Academy of Science.

Kuznetsov, V. A. and M. V. Volkenshtein. 1979. Dynamics of cellular immunological antitumor reactions. II. Qualitative analysis of the model (in Russian). In Mathematical Methods of Systems Theory, pp. 72-100. Frunze: Kirghiz State University.

Kuznetsov, V. A., V. P. Zhivoglyadov and L. A. Stepanova. 1993. Kinetic approach and estimation of parameters of cellular interaction between the immunity system and a tumor. Archiv. Immunol. Ther. Exp. 41, 21-32.

Lefever, R. and T. Erneaux. 1984. On the growth of cellular tissues under constant and fluctuating environmental conditions. In Nonlinear Electrodynamics in Biological Systems, P. Adley and A. F. Lowrence (Eds), pp. 287-305. New York and London: Plenum Press.

Lefever, R., J. Hiernaux, J. Urbain and P. Meyers. 1992. On the kinetics and optimal specificity of cytotoxic reactions mediated by T-lymphocyte clones. Bull. math. Biol. 54, 839-873.

Liu, Ch.-M., Y. Suzuki, L. Chen, T. Okayasu, C. E. Calkins and E. F. Wheelock. 1990. Maintenance and cure of the L5178 murine tumor dormant state by interleukin-2: In vivo and in vitro effects. Cancer Res. 50, 1361-1367.

Look, A. T., T. J. Schriber, J. F. Nawrocki and W. H. Murphy. 1981. Computer simulation of the cellular immune response to malignant lymphoid cells: Logic of approach, model design and laboratory verification. Immunol. 43, 677-690.

Macken, C. A. and A. S. Perelson. 1984. A multistage model for the action of cytotoxic T lymphocytes in multicellular conjugates. J. Immunol. 132, 1614-1624.

Mathe, G. and P. Rejzenstein. 1986. Managing minimal residual malignant disease. Oncology 43, 137-142.

Menta, B. C. and M. B. Agarwal. 1980. Cyclic oscillations in leukocyte count in chronic myeloid leukemia. Acta. Haematol. 63, 68-70.

Merrill, S. J. 1982. Foundations of the use of enzyme kinetic analogy in cell-mediated cytotoxicity. Math. Biosci. 62, 219-236.

Merrill, S. J. and S. Sathananthan. 1986. Approximate Michaelis-Menthen kinetics displayed in a stochastic model of cell-mediated cytotoxicity. Math. Biosci. 80, 223-238.

Mohler, R. R. and K. S. Lee. 1989. Dynamic analysis and control of cancer. In Int. Conf. IEEE Engng Med. Biol. Seattle, pp. 1-2. 
Nelson, D. S. and M. Nelson. 1987. Evasion of host defenses by tumors. Immunol. Cell. Biol. 65 , 287-304.

Old, L. J., E. A.Boyse, D. A. Clarke and F. A. Carswell. 1962. Antigenic properties of chemically induced tumors. Ann. N. Y. Acad. Sci. 101, 80-106.

Perelson, A. S. and G. I. Bell. 1982. Delivery of lethal hits by cytotoxic T lymphocytes in multicellular conjugates occurs sequentially but at random. J. Immunol. 129, 2796-2801.

Perelson, A. S. and C. A. Macken. 1984. Kinetics of cell-mediated cytotoxicity: Stochastic and deterministic multistage models. Math. Biosci. 170, 161-194.

Prehn, R. T. 1972. The immune reaction as a stimulator of tumor growth. Science 4031, 170-171.

Prehn, R. T. 1983. Review/commentary. The dose-response curve in tumor immunity. Int. J. Immunopharm. 5, 255-257.

Prigogine, I. and R. Lefever. 1980. Stability problems in cancer growth and nucleation. Comp. Biochem. Physiol. 67, 389-393.

Rescigno, A. and C. DeLisi. Immune surveillance and neoplasia. II. A two-stage mathematical model. Bull. math. Biol. 39, 487-497.

Reynolds, C. W., R. H. Wiltrout, S. Reichardi and R. B. Herberman. 1985. Measurements of the in vivo turnover rates of rat peripheral blood and spleen large granular lymphocytes. Natural Immun. Cell Growth Regul.9, 272.

Sampson, D., T. G. Peter, S. D. Lewis, J. Metzig and B. E. Murtz. 1977. Dose dependence of immunopotentiation and tumor regression induced by levamisole. Cancer Res. 37, 3526-3528.

Siu, H., E. S. Vitetta, R. D. May and I. W. Uhr. 1986. Tumor dormancy. I. Regression of BCL tumor and induction of a dormant tumor state in mice chimeric at the major histocompatibility complex. J. Immunol. 137, 1376-1382.

Slavin, S. and S. Strober. 1978. Spontaneous murine B-cell leukemia. Nature 272, 624-626.

Stewart, T. H. M. and E. F. Wheelock. 1992. Cellular Immune Mechanisms and Tumor Dormancy. Boca Raton, FL: CRC.

Strober, S., E. S. Gronowicz, M. R. Knapp and S. Slavin. 1979. Immunobiology of a spontaneous murine B cell Leukemia (BCL). Immunol. Rev. 48, 169-195.

Swan, G. W. 1977. Some Current Mathematical Topics in Cancer Research. Ann Arbor, MI: University Microfilms International.

Tanaka, K., T. Yoshioka, C. Bieberich and G. Jay. 1988. Role of the major histocompatibility complex class I antigens in tumor growth and metastasis. Ann. Rev. Immunol. 6, 359-380.

Thoma, J. A., G. J. Thoma and W. Clark. 1978. The efficiericy and linearity of the radiochromium release assay for cell-mediated cytotoxicity. Cell Immunol 40, 404-418.

Thorn, R. M. and C. S. Henney. 1976. Kinetic analysis of target cell destruction by effector T cell. J. Immunol. 117, 2213-2219.

Thorn, R. M. and C. S. Henney. 1977. Kinetic analysis of target cell destruction by effector cells. II. Changes in killer cell avidity as a function of time and dose. J. Immunol. 119, 1973-1978.

Umiel, T. and N. Trainin. 1974. Immunological enhancement of tumor growth by syngeneic thymus-derived lymphocytes. Transplant 18, 244-250.

Uhr, J. W., T. Tucker, R. D. May, H. Siu and E. S. Vitetta. 1991. Cancer dormancy: Studies of the murine $\mathrm{BCL}_{1}$ lymphoma. Cancer Res. (Suppl.) 51, 5045s-5053s.

Uyttenhove, C., J. Maryanski and T. Boon. 1983. Escape of mouse mastocytoma P815 after nearly complete rejection is due to antigen-loss variants rather than immunosuppression. $J$. Expl Med. 157, 1040-1052.

Weinhold, K. J., L. T. Goldstein and E. F. Wheelock. 1979a. The tumor dormant state. Quantitation of L5178Y cells and host immune response during the establishment. $J$. Expl Med. 149, 732-744.

Weinhold, K. J., D. A. Miller and E. F. Wheelock. 1979b. The tumor dormant state. Comparison of L5178Y cells used to establish dormancy with those that emerge after its termination. $J$. Expl Med. 149, 745-747.

Weiss, L., S. Morecki, E. S. Vitetta and S. Slavin. 1983. Suppression and elimination of BCL leukemia by allogeneic bone marrow transplantation. J. Immunol. 130, 2452-2455. 
Wheelock, E. F. and M. K. Robinson. 1983. Biology of disease. Endogenous control of the neoplastic process. Lab. Investigation 48, 120-139.

Wheelock, E. F., K. J. Weinhold and J. Levich. 1981. The tumor dormant state. Adv. Cancer Res. 34, 107-135.

Wiggins, S. 1990. Introduction to Applied Nonlinear Dynamical Systems and Chaos. New York, NY: Springer.

Yefenof, E., L. J. Picker, R. H. Scheuermann, T. F. Tucker, E. S. Vitetta and J. W. Uhr. 1993. Cancer dormancy: Isolation and characterization of dormant lymphoma cells. Proc. Natl Acad. Sci. USA 90, 1829-1833.

Yermakova, A., P. Valko and S. Vajda. 1982. Direct intergral method via spline approximation for estimating rate constant. Appl. Catalysis 2, 139-154.

Received 23 September 1992 